\title{
IMPROVED PROPRIOCEPTIVE FUNCTION BY APPLICATION OF SUBSENSORY ELECTRICAL NOISE: EFFECTS OF AGING AND TASK-DEMAND
}

\author{
DIANA R. TOLEDO, ${ }^{\mathrm{a} *}$ JOSÉ A. BARELA $^{\mathrm{b}, \mathrm{c}}$ AND \\ ANDRÉ F. KOHN \\ ${ }^{a}$ Neuroscience Program and Biomedical Engineering Laboratory, \\ University of São Paulo, EP-USP, PTC, São Paulo, Brazil \\ ${ }^{\mathrm{b}}$ Institute of Physical Activity and Sport Sciences and Graduate \\ Program in Human Movement Sciences, Cruzeiro do Sul University, \\ São Paulo, Brazil \\ 'Institute of Bioscience, São Paulo State University, Rio Claro, Brazil
}

is a determining factor for sensorimotor improvements due to electrical noise stimulation. (c) 2017 IBRO. Published by Elsevier Ltd. All rights reserved.

Key words: aging, proprioception, electrical noise, stochastic resonance, posture.

\section{INTRODUCTION}

over the lower limbs has been shown to improve proprioception and postural control under certain conditions. Whereas the effect specificity seems to depend on several factors, studies are still needed to determine the appropriate method for training and rehabilitation purposes. In the current study, we investigated whether the application of subsensory electrical noise over the legs improves proprioceptive function in young and older adults. We aimed to provide evidence that stronger and age-related differential effects occur in more demanding tasks. Proprioceptive function was initially assessed by testing the detection of passive ankle movement (kinesthetic perception) in twenty-eight subjects (14 young and 14 older adults). Thereafter, postural control was assessed during tasks with different sensory challenges: i) by removing visual information (eyes closed) and; ii) by moving the visual scene (moving room paradigm). Tests performed with the application of electrical noise stimulation were compared to those performed without noise. The results showed that electrical noise applied over the legs led to a reduction in the response time to kinesthetic perception in both young and older adults. On the other hand, the magnitude of postural sway was reduced by noise stimulation only during a more challenging task, namely, when the optical flow was changing in an unpredictable (nonperiodic) manner. No differential effects of stimulation between groups were observed. These findings suggest that the relevance of proprioceptive inputs in tasks with different challenges, but not the subjects' age,

\footnotetext{
${ }^{*}$ Corresponding author at: Biomedical Engineering Laboratory, University of São Paulo, EP-USP, PTC, Avenida Professor Luciano Gualberto, Travessa 3, n. 158, São Paulo, SP 05508-900, Brazil. E-mail address: dianatoledo12@hotmail.com (D. R. Toledo).

Abbreviations: ANOVA, analysis of variance; AP, anterior-posterior; $\mathrm{CL}$, confidence level; $\mathrm{CM}$, complex movement of the room; $\mathrm{EC}$, eyes closed; ES, condition with electrical noise stimulation; MANOVA, multivariate analysis of variance; ML, medial-lateral; MSA, mean sway amplitude; MSV, mean sway velocity; NES, condition without electrical noise stimulation; OA, older adults; PFTV, position of the fifth thoracic vertebra; RT, response time; SM, simple movement of the room; SR, stochastic resonance; ST, sensory threshold; YA, young adults.
}

Falls in older adults represent a major public health problem with serious consequences. Besides carrying high costs with health care utilization and institutionalization, physical injuries due to falls result in several secondary consequences such as reduced mobility, caused either by the fall-related injuries themselves or by fear of falling. Despite perceiving themselves at higher risk of falls, older adults are often reluctant to adhere to interventions and to use assistive devices (Aminzadeh and Edwards, 1998). Therefore, appropriate solutions for improving sensorimotor function need to be implemented in order to prevent falls and promote welfare in fragile individuals, especially those who resist participating in preventive and rehabilitation programs.

Falls can be avoided by efficient postural actions, which depend on complex interactions between environmental constraints, task demands and motor control in order to keep the center of mass within the base of support. Balance and gait impairments, in addition to sensory dysfunction, weakness, cognitive deficits and use of specific medications are the main intrinsic factors that increase the risk of falls in older adults (Tinetti and Kumar, 2010). However, the severity of the risk factors many times is only identified after at least one fall, increasing the probability of further falls (Tinetti and Kumar, 2010). Thus, the identification and treatment of the risk factors in early stages may result in a significant impact for fall prevention.

Postural control involves an intricate relationship between sensory information and muscle activity and can be investigated by manipulating sensory cues and observing the corresponding postural responses. For instance, the unavailability of visual cues (i.e., eyes closed) already destabilize upright stance, especially for older adults, although age-related postural control changes are more evident in more demanding tasks 
(Prioli et al., 2006; Toledo and Barela, 2014). Moreover, a sensory conflict situation generated by the moving room paradigm was shown to be a sensitive method for detecting postural control impairments in older adults (Prioli et al., 2006; Toledo and Barela, 2014). In this paradigm, the visual field movement has a destabilizing effect due to the sensory conflict situation (i.e., visual cues indicate that a movement is occurring whereas somatosensory and vestibular cues do not).

In order to resolve a sensory conflict situation, the postural control system must constantly reweight the importance of the available sensory cues (Jeka et al., 2008; Barela et al., 2013; Genoves et al., 2016) a functional process that is impaired in older adults (Prioli et al., 2006; Barela et al., 2013; Toledo and Barela, 2014), predisposing them to increased instability and falls. Actually, worse postural performance in the moving room situation was related to reduced kinesthetic perception at the ankle joint (Toledo and Barela, 2014). Proprioceptive impairment in the lower limbs has been considered, among other sensory changes occurring due to aging, the main contributor to balance loss in older adults (Fitzpatrick and McCloskey, 1994; Lord and Ward, 1994; McChesney and Woollacott, 2000; Toledo and Barela, 2014).

These findings indicate the need to uncover methods that would improve somatosensory lower limb functioning. An emerging approach has shown that joint proprioception can be improved when noisy stimulation is applied over the limbs (Collins et al., 2009; RibotCiscar et al., 2013). The premise is that the detection, transmission and discrimination of sensory signals are enhanced by the addition of mechanical or electrical noise, a phenomenon known as stochastic resonance (SR) (Collins et al., 1996; Moss et al., 2004; McDonnell and Abbott, 2009). Application of an optimal noise level is suggested to lead to appropriate small amplitude fluctuations in transmembrane potentials, bringing neurons to reach threshold even to an initial subthreshold stimuli (Volgushev and Eysel, 2000). Stochastic resonance associated with sensory function may yield global changes in the motor control system. i.e., reduced postural sway in adults (Gravelle et al., 2002; Priplata et al., 2002, 2003, 2006; Magalhaes and Kohn, 2011, 2012, 2014; Kimura and Kouzaki, 2013; Lipsitz et al., 2015). Importantly, postural sway reductions during noisy stimulation were also observed in individuals with sensorimotor impairments, such as patients after stoke (Priplata et al., 2006), individuals with ankle instability (Ross et al., 2007) and in older adults subjected to cutaneous stimulation of the foot soles (Priplata et al., 2003; Dettmer et al., 2015; Lipsitz et al., 2015).

Despite some studies that have shown that the application of noise over the limbs improves the functioning of the proprioceptive system specifically (Collins et al., 2009; Ribot-Ciscar et al., 2013), such effects have not yet been reported for older adults. Thus, the aim of the present study was to investigate the effect of subsensory electrical noise on kinesthetic lower limb perception in older adults in comparison to young adults.
Moreover, we investigated whether the same stimulation parameters used during the kinesthetic perception assessment can induce postural improvements during conditions of visual input manipulation (eyes closed and moving room condition). Whereas recent studies with vibratory noise have shown that SR effects in reducing postural sway are more expressive in older versus young adults (Priplata et al., 2002; Wu et al., 2007; Dettmer et al., 2015) differential effects seem to be dependent on the task demand. For instance, during a sensory conflict task (i.e., moving visual surrounding), addition of mechanical noise improved postural control in older adults (Dettmer et al., 2015) but no effects were observed in young adults (Keshner et al., 2014; Dettmer et al., 2015). On the other hand, no effects of SR mechanical stimulation on postural control were obtained during translation of support surface in both young and older adults (Borel and Ribot-Ciscar, 2016; Dettmer et al., 2016). Considering that SR effects on motor control still need to be further understood, the goal of the present research was to verify if there are age-related differential SR effects when electrical noise is applied during tasks with different sensory (visual) demands. The efficacy of electrical noise stimulation on sensorimotor functioning investigated in the current study provides data that help in the selection of the most appropriate stimulation technique (electrical or mechanical) for rehabilitation purposes, based on the intended effects.

In the current study, we investigated the effect of SR on ankle proprioceptive function in situations with different task demands. In a first scenario, proprioceptive function was assessed isolated from other systems, by testing the detection of passive ankle movement (kinesthetic perception). Thereafter, SR effects were investigated during postural tasks involving reweighting processes, in which the importance of the somatosensory system was upweighted, either by removing visual information (eyes closed) or by moving the visual scenario (moving room paradigm). The movement of the room occurred in two different ways: simple (single frequency) and complex (sum of three frequencies resulting in a nonperiodic movement). Complex room movement conditions further challenge posture control function because more accurate sensory signals are required from the ankle joint for both older and young adults (Toledo and Barela, 2014). Whereas individuals with nearly optimum baseline performance could not benefit from SR stimulation (Priplata et al., 2006; Dettmer et al., 2015), stochastic electrical stimulation applied on the lower limbs is expected to improve postural control in young adults only for the more demanding task (i.e., complex room movement). On the other hand, we hypothesize that postural control in older subjects will be susceptible to the stimulation effects even for less demanding tasks, (i.e., eyes closed and simple room movement). Regarding the SR effects on the response time to kinesthetic perception, older adults are expected to benefit more than young adults from the electrical stimulation. 


\section{EXPERIMENTAL PROCEDURES}

\section{Participants}

Fourteen healthy community-dwelling older adults (70.68 \pm 4.72 years) (OA) and 14 young adults (29.79 \pm 2.12 years) (YA) adults (9 females in each group) participated in this study. The older participants were chosen among those who had not been enrolled in any regular physical activity program (for at least three years), had not suffered any falls in the last two years, had no vestibular impairment, pain, visual, osteomuscular and/or neurological disorders, and had not made use of benzodiazepine or antidepressant drugs. All subjects were right-footed, where footedness was self-reported as to which foot would be chosen for kicking a ball and was confirmed by the observation of the foot chosen to step on the weighing scale.

\section{Procedures}

This study was conducted in accordance with the Declaration of Helsinki and its procedures were approved by the Institutional Ethics Committee. In addition, all procedures were carried out with adequate understanding and written consent from each participant.

\section{Electrical stimulation}

The electrical stimulation consisted of a zero-mean Gaussian bandpass filtered noise with a bandwidth from 5 to $1,500 \mathrm{~Hz}$ (Fig. 1A shows a sample time series and Fig. 1B shows the corresponding power spectral density), generated via a LabView-based system
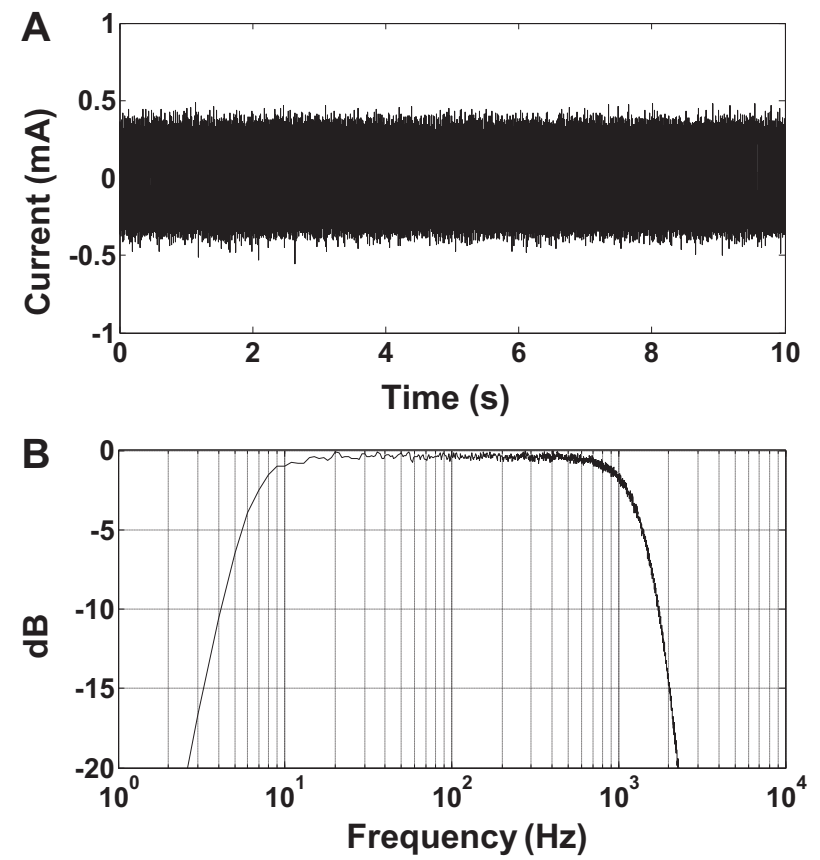

Fig. 1. Representative sample time series (A) and corresponding power spectrum (B) of the bandpass filtered white noise current signal applied to the subject's leg with intensity set at $90 \%$ of the sensory threshold level.
(National Instruments, USA) and applied to the subject through two STMISOL stimulus isolation units (BIOPAC Systems, Inc.), one for each leg. Pairs of flexible siliconstimulating electrodes $(12 \times 7 \mathrm{~cm})$ were fixed bilaterally over the posterior side of the subjects' legs. The proximal electrode was positioned midway just below the inferior margin of the two heads of the gastrocnemius muscles. The distal electrode was placed $3 \mathrm{~cm}$ below the proximal electrode.

An intensity of stimulation was pre-defined in the Labview software. The generated signal was transmitted to a potentiometer box, which permitted individual adjustments (by means of knobs) of the intensity of stimulation to be applied on each leg. The stimulus signals were then transmitted from the potentiometer box to the STIMSOL units and then to the electrodes. For the definition of the stimulation intensity to be used for a given subject, a clearly perceptible stimulus (maximal RMS of stimulation varying between $5 \mathrm{~mA}$ and $8 \mathrm{~mA}$ ) was initially applied to one leg during $10 \mathrm{~s}$. Then, the stimulus was applied to the other leg and the subject reported whether the intensity of stimulation was similar between the legs. Intensity adjustments (by means of the potentiometer box) were made until the intensities were similarly perceived in both legs. Progressively weaker stimuli were then applied until both were below perception. Sensory threshold (ST) was defined as the highest intensity of electrical noise stimulation that the subject was unable to perceive (for at least three stimulation trials). Increments and decrements around the highest unperceivable intensity were performed in order to confirm the ST. The ST was individually obtained for each subject and for each assessment (one intensity for kinesthetic perception and one intensity for posture control). For the assessment protocols, the stimulus intensity was individually set at $90 \%$ of the respective ST. At this stimulation intensity, the subjects were not aware of the treatment condition and no electrically induced muscle contractions occurred. The choice of the intensity level at $90 \%$ ST was based on a previous study (Magalhaes and Kohn, 2012) which indicated that electrical noise stimulation at this intensity was effective in decreasing postural sway in comparison to the control condition. The intensity of stimulation was the same throughout each specific protocol. The subject was asked periodically if he had felt any kind of electrical stimuli and the answer was invariably no. Since the electrical current level applied to the subjects could not be measured during the original experiments due to the analog hardware method of final intensity adjustments (made through knobs), extra experiments were posteriorly performed with a sample of 10 agematched $(\mathrm{YA}=30.00 \pm 4.80$ years old; $\mathrm{OA}=69.00$ \pm 3.94 years old) subjects for estimating the stimulation level ranges corresponding to the present experimental protocol. The sensory threshold for young adults was obtained for RMS current value equal to $1.72 \mathrm{~mA}$ $( \pm 0.27, N=5)$ and for older adults $1.11 \mathrm{~mA}( \pm 0.33$, $N=5$ ). The individual $S T$ values (in $\mathrm{mA}$ ) obtained, in ascending order, were: $1.45 ; 1.50 ; 1.70 ; 1.85 ; 2.10$ for YA and $0.70 ; 0.95 ; 1.00 ; 1.40 ; 1.50$ for OA. 


\section{Kinesthetic perception assessment}

The assessment of kinesthetic perception was performed similar to our previous studies (Toledo and Barela, 2014; Toledo et al., 2016a). Briefly, participants were seated with knees maintained at $90^{\circ}$ and trunk at approximately $120^{\circ}$ (Fig. 2). Their right foot rested on a pedal, which was passively dorsiflexed at $0.5^{\circ} \mathrm{s}$ for $5 \mathrm{~s}$, from a starting ankle angle set at $90^{\circ}$, and then returned to the basal angle with similar speed and duration (i.e., total movement last for $10 \mathrm{~s}$ ). Participants were instructed to keep their eyes closed and press a button with their right thumb as fast as possible whenever the ankle dorsiflexion movement onset was detected. Passive movement was performed at random intervals, starting between 3 and $9 \mathrm{~s}$ after a verbal preparatory signal. Five 4-min trials were performed with 2-min rest in between, totaling 60 movements. Out of 60 movements, 30 were performed with electrical noise stimulation (ES condition), and 30 without electrical stimulation (NES condition), randomly distributed in each trial. The electrical stimulation lasted $7 \mathrm{~s}$, starting $2 \mathrm{~s}$ before the ankle movement onset and ending when the movement direction inverted to plantarflexion. The pedal's movement was produced by a system consisting of a servo-motor (AC Brushless Servo-Motor, Model SWA-40-1.6-30), a reducer (Dynabox Medium - France) and servo electronics (Weg - Brazil). The analog signal to command the servo-controller was generated via Labview software (National Instruments, USA). The pedal's movement was registered using IRED emitters of an Optotrak system (Certus, NDI, Inc.) and the position signal was acquired at a sampling frequency of $100 \mathrm{~Hz}$.

\section{Postural control assessments}

Postural control was assessed in three conditions, in the following order: i) eyes closed (EC); ii) eyes open with simple movement of the room (SM); and iii) eyes open with complex movement of the room (CM) (Fig. 2). For the EC condition, each trial lasted $30 \mathrm{~s}$ whereas for the $\mathrm{SM}$ and $\mathrm{CM}$ conditions, each trial lasted $60 \mathrm{~s}$. Six trials
KINESTHETIC PERCEPTION

Fig. 2. Schematic representation of the experimental protocol. Firstly, the proprioceptive system was tested: the subject was seated and pressed a button with his right hand when he felt his right foot moving. Thereafter, postural control was tested under eyes closed with no room movement (EC), and under eyes open during simple and complex movements of the room (SM and CM respectively). Room movement waveforms for SM and CM conditions are displaced at the bottom. All assessments were performed both with and without electrical noise stimulation.

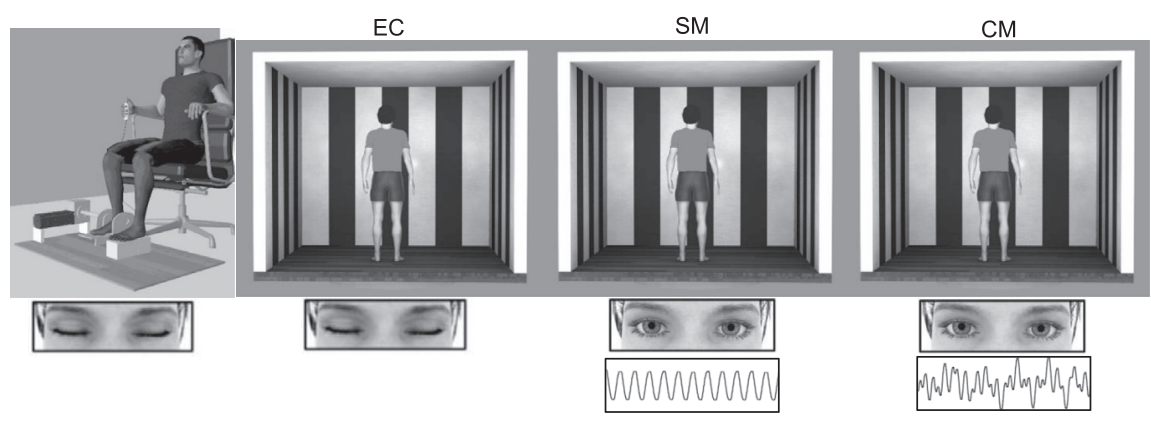

were performed for each condition (3 ES and 3 NES), randomly distributed. For the ES condition, the electrical stimulation was applied during the whole trial. Participants stood barefoot with feet apart at shoulder width and were asked to stand, as still as possible, inside a moving room. For the SM and CM conditions, participants were also instructed to keep looking at a target (white circle, $d=5 \mathrm{~cm}$ ) placed 1 meter away at eye level. The moving room consisted of three walls and a ceiling $(2 \mathrm{~m}$ long $\times 2 \mathrm{~m}$ wide $\times 2 \mathrm{~m}$ high), with wheels placed on linear rails so that it could be moved backward and forward (walls and ceiling moved, the floor did not). The walls were an alternation of white (42 cm wide) and black (22 cm wide) vertical stripes, which enhanced the contrast of the surround visual information. Two 20-watt triple tube compact fluorescent light bulbs were placed horizontally on the backside of the ceiling and directed to the front wall to maintain the same luminosity throughout the data collection. The room was moved by a servomotor system composed of a linear guide (Ottime, model PL6-90C-LD-MT-RC), a stepper motor (Ottime, model SM3452808), and a motor driver (Ottime, model MBD-8080DC) and controlled by a specific software (Motion Planner 4.3). In the SM condition, the room movement consisted of a pure sinusoidal motion with a frequency of $0.2 \mathrm{~Hz}$, peak-topeak displacement of $1.1 \mathrm{~cm}$ and peak velocity of $0.69 \mathrm{~cm} / \mathrm{s}$ (Fig. 3). In the CM condition, the room movement consisted of a sum of three sinusoids, with frequencies of $0.1,0.3$ and $0.47 \mathrm{~Hz}$ and peak-to-peak displacement of $3.8 \mathrm{~cm}$. These movement parameters were based on a previous study that showed differences in the postural control between young and older adults in these conditions (Toledo and Barela, 2014). Body sway and moving room displacements were obtained using an Optotrak system with an IRED marker measuring the position of the fifth thoracic vertebra (PFTV), and another IRED marker placed at the frontal wall. The marker position signals were acquired at a sampling frequency of $100 \mathrm{~Hz}$. For both body sway and moving room data, a low-pass filter was applied ( 5 and $10 \mathrm{~Hz}$, respectively).

\section{Data analyses}

For the assessment of kinesthetic perception, the response time (RT) was calculated as the temporal difference between the movement onset and the signal generated by button pressing. Responses that took longer than $4 \mathrm{~s}$ to occur were discarded from the analysis.

For the postural control assessment in EC condition, trajectory, mean sway velocity (MSV) and mean body sway amplitude (MSA) were computed as dependent variables, the last two being calculated in both anterior-posterior (AP) and medial-lateral (ML) directions. Trajectory (actually, trajectory length) was estimated 

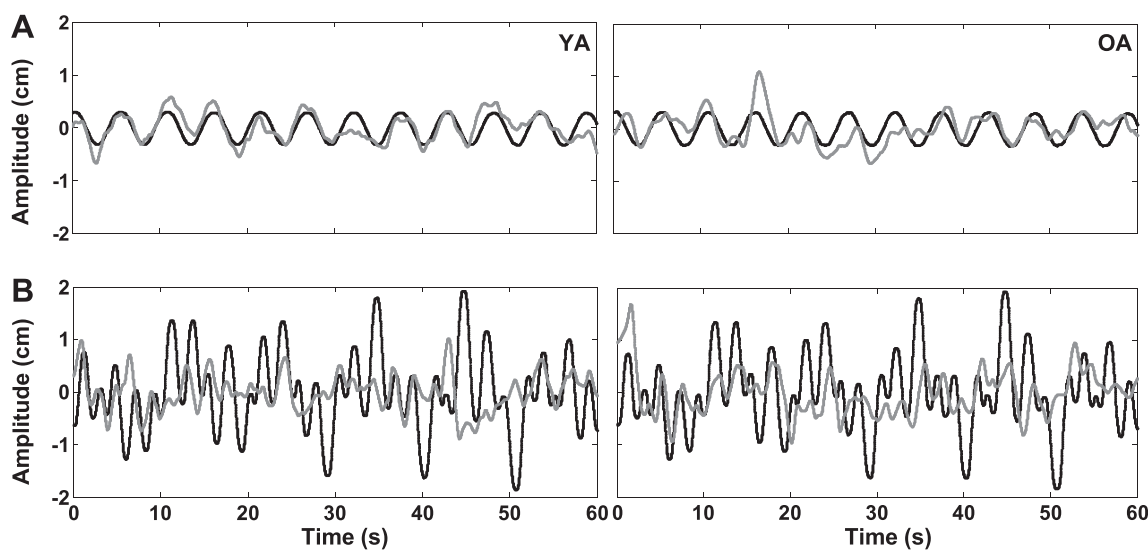

Fig. 3. Sample time series of the moving room (black line) and body sway (gray line) for both young $(\mathrm{YA})$ and older $(\mathrm{OA})$ adults with no electrical stimulation: simple $(\mathrm{A})$ and complex $(\mathrm{B})$ room movement conditions.

using the Pythagorean theorem applied to the samples of PFTV in the AP and ML directions. MSA was calculated as the standard deviation of the body sway signal (PFTV) in the AP and ML directions during the whole trial. MSV was computed as the mean of the absolute values of the instantaneous velocities (computed by the differences between adjacent samples of the respective PFTV signals) in the AP and ML directions. For the trials in which the room was moved (SM and CM), MSA, coherence and gain were also computed, the last two being explained in what follows. Coherence indicated the coupling strength in the frequency domain between the visual input (modulation of the optic flow by the moving room) and body sway. Coherence (or magnitude-squared-coherence) was computed as the ratio of the absolute value squared of the crossspectrum of the two signals (body and room sway) and the product of the auto-spectra of body sway and room sway. Coherence values were considered to be significant if they were above the confidence level $(C L)$ (Rosenberg et al., 1998) given below:

$C L(\alpha)=1-(1-\alpha)^{1 /(n-1)}$,

where $n$ is the number of segments used in the computation of the spectra that result in the coherence function, and $\alpha$ is the desired level of confidence (0.05). For the coherence analysis, the signals recorded from three trials in each condition were concatenated, creating a 180-s data series and then, divided into 18 segments of 1-s duration, allowing a frequency resolution of $0.1 \mathrm{~Hz}$. For each subject, the $\mathrm{CL}$ was 0.016 . For the SM condition, the only frequency of interest in the coherence analysis was $0.2 \mathrm{~Hz}$, whereas for the $\mathrm{CM}$ conditions the coherence analysis focused on three frequencies $(0.1,0.3$ and $0.5 \mathrm{~Hz})$. The frequency of $0.5 \mathrm{~Hz}$ in the analyses of coherence and gain (see below) was chosen as being the closest frequency to the $0.47-\mathrm{Hz}$ component of the optic flow input, due the $0.1-\mathrm{Hz}$ resolution of the analyses. A coherence value close to one at a given frequency indicates a strong association between the two signals at that frequency.
Conversely, coherence values close to zero indicate independence between them.

Gain was defined as the ratio between body sway amplitude (PFTV) in the AP direction and room movement amplitude at a frequency of room movement. It quantifies the influence of visual manipulation provided by the moving room on body sway. Similarly to coherence calculations, gain values were also calculated for only one frequency $(0.2 \mathrm{~Hz})$ for the SM condition, and for three frequencies $(0.1,0.3$ and $0.5 \mathrm{~Hz}$ ) for the $\mathrm{CM}$ condition. Gain values smaller/larger than one indicate low-/high-induced body sway due to visual manipulation.

All data processing and variable calculation were performed through custom routines written in MATLAB (Math Work Inc., USA).

\section{Statistical analyses}

As normality and homogeneity assumptions were accepted by appropriate tests, analysis of variance (ANOVA) and multivariate analyses of variance (MANOVAs) were conducted. A one-way MANOVA was conducted to test whether the participants' height and the weight were similar between groups. For the remaining analyses, groups (YA and $\mathrm{OA}$ ) and conditions (NES and ES) were the factors, the latter being treated as a repeated measure.

For the proprioceptive assessment, a two-way ANOVA was initially conducted for comparison of the number of valid trials obtained for each group and condition. Another two-way ANOVA was conducted to compare the RT between the independent variables. This analysis tested the hypothesis that both young and older adults would improve the RT with the application of noise, with older adults improving significantly more than young adults.

For the postural control assessment in the EC condition, a two-way ANOVA was performed for trajectory. Two additional two-way MANOVAs were conducted for the following dependent variables: MSA AP and MSA ML for the first MANOVA and MSV AP and MSV ML for the second MANOVA. For the SM condition three two-way ANOVAs were performed, one for each dependent variable (MSA in the AP direction, gain and coherence). Finally, for the CM condition, a two-way ANOVA was conducted for the dependent variable $M S A$ in the AP direction. Additional two MANOVAs were conducted, being the first for the gain values at $0.1,0.3$ and $0.5 \mathrm{~Hz}$ as the dependent variables and the second for the coherence values at 0.3 and $0.5 \mathrm{~Hz}$ as the dependent variables. Coherence values at $0.1 \mathrm{~Hz}$ were not included in the statistical analyses since a weak coupling between signals was observed at this frequency (see Results session). We hypothesized that postural sway would be reduced by 
the application of noise in all the conditions for the older group, whereas the young group would present a reduction only for the $\mathrm{CM}$ condition. For each analysis, partial eta-squared $\left(\eta_{\mathrm{p}}^{2}\right)$ indicated the effect size, which was considered high when the index was higher than 0.14 (Tabachnick and Fidell, 2007). When necessary, Tukey's HSD post hoc tests were conducted.

In order to investigate the relationship between proprioceptive and postural control improvements with the application of noise stimulation, Pearson's correlation coefficients were computed between RT and postural control variables that differed between NES and ES. For the correlation analyses, the percentage of change of the variables in the ES condition with respect to the NES condition was used.

All analyses were performed using the software package SPSS (SPSS 15.0 for Windows), with statistical significance set at $P<0.05$.

\section{RESULTS}

\section{Anthropometric measures}

The average height and weight of the young participants were $1.65( \pm 0.08) \mathrm{m}$ and $64.73 \quad( \pm 12.24) \mathrm{kg}$, respectively. For the elderly group, the average height and weight of the participants were $1.63( \pm 0.9) \mathrm{m}$ and $65.89( \pm 11.44) \mathrm{kg}$, respectively. Anthropometric measures were not statistically different between groups $(P>0.05)$.

\section{Kinesthetic perception}

The mean number of valid trials for the YA group was 24 $( \pm 8)$ for both NES and ES conditions. For the OA group, $24( \pm 6)$ valid trails were obtained for both NES and ES conditions. The number of valid responses did not differ statistically between groups and conditions and no interaction was obtained between groups $(P>0.05)$.

The mean response times for the young group were $1.41( \pm 0.26) \mathrm{s}$ and $1.37( \pm 0.31) \mathrm{s}$ for the NES and ES conditions, respectively. For the older group the mean response times were $2.15( \pm 0.47) \mathrm{s}$ and $2.03( \pm 0.42)$ $\mathrm{s}$ for the NES and ES conditions, respectively, i.e., a $120-\mathrm{ms}$ decrease in the average. The statistical values $\left(\mathrm{P}, \mathrm{F}\right.$ and $\eta_{\mathrm{p}}^{2}$ ) obtained from ANOVA are shown in Table 1. The analyses showed that RT was reduced when the electrical noise was applied and was shorter for the young as compared to the older group.

\section{Postural control}

Fig. 4 depicts the mean values of the variables obtained for each group in each stimulation condition for the EC condition. The respective statistical values obtained from ANOVA and MANOVAs are shown in Table 1. ANOVA revealed significant interaction between the group and condition factors for trajectory. Post Hoc analyses revealed that the trajectory was larger for the OA group only for the NES condition. For the MSA variable, no differences were obtained between group and stimulation condition factors. In addition, no interaction between the factors was obtained. On the other hand, for the MSV variable, despite significant main effects were not obtained for both group and stimulation condition factors, significant interaction between group and condition was found. Univariate analyses showed that the interaction was significant only for the AP direction. Post hoc analyses revealed that the MSV AP was higher for the OA group only for the NES condition.

The results obtained for MSA, gain and coherence variables for the SM condition are shown in Fig. 5. All subjects showed coherence values above the $\mathrm{CL}$ (0.016) and the analyses revealed no significant differences between the groups for the variables analyzed. Also, no differences were found between the stimulation conditions. In addition, no interaction between the factors was found. The statistical results are shown in Table 1.

The results obtained for the $\mathrm{CM}$ condition are depicted in Fig. 6 and Table 1. The analyses showed that the sway amplitude (MSA) was reduced in both age groups when the electrical noise was applied. Moreover, body sway was lower for the young as compared to the older group. For the gain variable, no differences were obtained for both group and condition factors and no interaction between factors was obtained. The results of the coherence analysis showed that only two subjects in each group presented values above the $\mathrm{CL}$ for the lowest frequency $(0.1 \mathrm{~Hz})$. On the other hand, 12 young and 10 older adults showed significant coherence for both $0.3-\mathrm{Hz}$ and $0.5-\mathrm{Hz}$ frequencies. Similar to the gain results, no significance was obtained for both group and condition factors and interaction between these factors.

\section{Correlation between proprioceptive and balance assessments}

No significant correlation between MSA (CM condition) and RT was observed $(P>0.05)$.

\section{DISCUSSION}

This study aimed to investigate whether the application of subsensory electrical noise over the posterior region of the legs improves kinesthetic function, leading to a reduction in the threshold for perception of ankle passive motion and an improvement in postural control performance in young and older adults. The results showed that the application of noise improved both kinesthetic perception and postural assessments, the latter being dependent on the task demand. Despite no significant differential effects of ES stimulation were obtained between age groups, older adults tended to benefit more from the electrical stimulation than young adults.

\section{Effect of SR stimulation on kinesthetic perception}

Considering that the level of noise applied over the limbs was subsensory, cognitive influences on the response time to kinesthetic perception can be ruled out. Alternatively, recruitment of afferent receptors that were initially non-responsive to passive movement is 
Table 1. Statistical values obtained for the assessments of kinesthetic perception assessment and postural control

\begin{tabular}{|c|c|c|c|c|c|}
\hline & & & Group & Condition & Group vs. Condition \\
\hline \multirow[t]{3}{*}{ Kinesthetic perception } & Response Time & $P$ & $<0.0001^{*}$ & $0.047^{*}$ & 0.300 \\
\hline & & $F(1,26)$ & 26.494 & 4.357 & 1.106 \\
\hline & & $\eta_{\mathrm{p}}^{2}$ & 0.505 & 0.144 & - \\
\hline \multirow{15}{*}{$\begin{array}{l}\text { Postural Control } \\
\text { EC }\end{array}$} & Trajectory & $P$ & 0.505 & 0.571 & $0.020^{*}$ \\
\hline & & $F(1,26)$ & 0.457 & 0.329 & 6.192 \\
\hline & & $\eta_{\mathrm{p}}^{2}$ & - & - & 0.192 \\
\hline & MSA & $P$ & 0.870 & 0.472 & 0.423 \\
\hline & & $F(2,25)$ & 0.140 & 0.773 & 0.882 \\
\hline & & $\eta_{\mathrm{p}}^{2}$ & - & - & - \\
\hline & MSV (Multivariate) & $P$ & 0.677 & 0.663 & $0.046^{*}$ \\
\hline & & $F(2,25)$ & 0.396 & 0.418 & 3.493 \\
\hline & & $\eta_{\mathrm{p}}^{2}$ & - & - & 0.218 \\
\hline & MSV AP (Univariate) & $P$ & - & - & $0.015^{*}$ \\
\hline & & $F(1,26)$ & - & - & 6.757 \\
\hline & & $\eta_{\mathrm{p}}^{2}$ & - & - & 0.206 \\
\hline & MSV ML (Univariate) & $P$ & - & - & 0.122 \\
\hline & & $F(1,26)$ & - & - & 2.560 \\
\hline & & $\eta_{\mathrm{p}}^{2}$ & - & - & - \\
\hline \multirow{10}{*}{$\begin{array}{l}\text { Postural Control } \\
\text { SM }\end{array}$} & MSA & $P$ & 0.698 & 0.647 & 0.573 \\
\hline & & & & & \\
\hline & & $F(1,26)$ & 0.154 & 0.214 & 0.325 \\
\hline & & $\eta_{\mathrm{p}}^{2}$ & - & - & - \\
\hline & Gain & $P$ & 0.222 & 0.125 & 0.351 \\
\hline & & $F(2,25)$ & 1.565 & 2.509 & 0.903 \\
\hline & & $\eta_{\mathrm{p}}^{2}$ & - & - & - \\
\hline & Coherence & $P$ & 0.059 & 0.147 & 0.690 \\
\hline & & $F(2,25)$ & 3.899 & 2.240 & 0.163 \\
\hline & & $\eta_{\mathrm{p}}^{2}$ & - & - & - \\
\hline \multirow{9}{*}{$\begin{array}{l}\text { Postural Control } \\
\text { CM }\end{array}$} & MSA & $P$ & $0.027^{*}$ & $0.028^{*}$ & 0.128 \\
\hline & & $F(1,26)$ & 5.459 & 5.426 & 2.472 \\
\hline & & $\eta_{\mathrm{p}}^{2}$ & 0.174 & 0.173 & - \\
\hline & Gain & $P$ & 0.137 & 0.159 & 0.581 \\
\hline & & $F(3,24)$ & 2.025 & 1.884 & 0.667 \\
\hline & & $\eta_{\mathrm{p}}^{2}$ & - & - & - \\
\hline & Coherence & $P$ & 0.578 & 0.534 & 0.393 \\
\hline & & $F(2,19)$ & 0.564 & 0.982 & 2.057 \\
\hline & & $\eta_{\mathrm{p}}^{2}$ & - & - & - \\
\hline
\end{tabular}

* Difference statistically significant.

postulated as one contributor mechanism for the increased perceptual sensitivity and reduced response time during electrical noise stimulation. Prior studies in humans provided evidence that mechanical noise changes the encoding of sensory receptors (Cordo et al., 1996; Ribot-Ciscar et al., 2013). Using microneurography, Ribot-Ciscar et al. (2013) showed that the activity of muscular spindles was triggered or improved when mechanical noise was applied to the ankle tendons during slow imposed ankle movement. None of the cutaneous receptors identified by this method responded to movement and noise. In addition, subjects enhanced the ability to detect the direction of the ankle movements imposed with a $0.04 \%$ selocity. We suggest that similar neurophysiological mechanisms may explain our findings. However, since we used electrical stimulation, we cannot assure that muscular receptors were selectively recruited with the application of noise and no cutaneous sensors contributed to the perceptual sensitivity enhancement. Furthermore, besides peripheral mechanisms, higher centers of the nervous system could also be involved. This assumption is based on studies showing a cross-modal SR effect on psychophysical and electrophysiological experiments, which hypothesize that initial subthreshold neurons located at brain regions can exhibit firing activity once the noise enters in the periphery (Manjarrez et al., 2007; Lugo et al., 2008; MendezBalbuena et al., 2015). Accordingly, projections between unimodal and multimodal brain areas would facilitate the SR effect to be transmitted from one sensory modality to another modality (Mendez-Balbuena et al., 2015). Based on this assumption, the effects of electrical noise stimulation on different receptors and axons could have propagated to higher levels of the nervous system and 

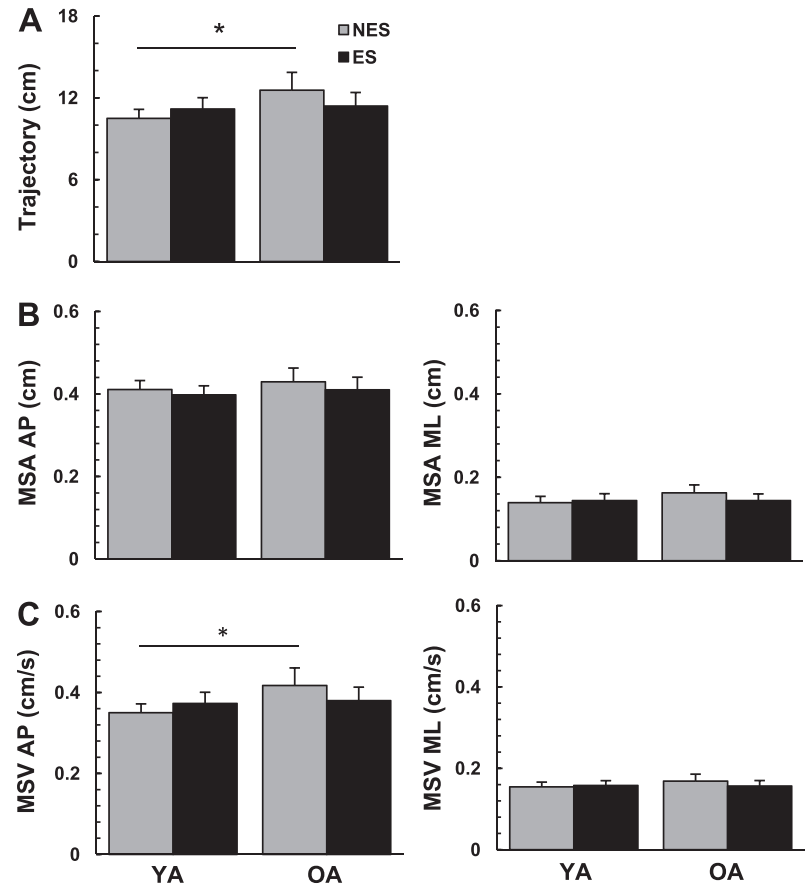

Fig. 4. Means and standard errors of postural control variables assessed during the Eyes Closed condition. Data are presented for both age groups (YA - young adults, OA - older adults) and both conditions (NES - No Electrical Stimulation, ES - Electrical Stimulation). *Significant differences between the age groups for the NES condition $(P<0.05)$. MSA AP - Mean sway amplitude in the anterior-posterior direction; MSA ML - Mean sway amplitude in the medial-lateral direction; MSV AP - Mean sway velocity in the anterior-posterior direction; MSV ML - Mean sway velocity in the medial-lateral direction.

activated neurons located at the brainstem or cortical areas involved in processing proprioceptive (and cutaneous) information.

\section{Similar SR effects between young and older adults during proprioceptive assessment}

The longer response time to perception of slow passive ankle movement in aged individuals confirms the results obtained in our previous studies (Toledo and Barela, 2010, 2014; Toledo et al., 2016a). One novel finding from the current study refers to the effect of the external subsensory electrical noise on the kinesthetic perception, which led to a reduction of the response time in both groups. Contrary to the expectations, no age-related differential effects of SR stimulation were observed. Perhaps an optimal stimulation intensity for each subject could lead to statistically significant differential effects between young and older subjects as SR phenomena in sensorimotor systems is characterized by an inverted U-like graph of the performance as a function of noise intensity (Magalhaes and Kohn, 2011; MendezBalbuena et al., 2012; Trenado et al., 2014).

Focusing on a putative application of noise-enhanced sensorimotor function for older subjects or specific patients, it would be interesting to investigate if indeed the efficacy would increase if the optimal intensity of
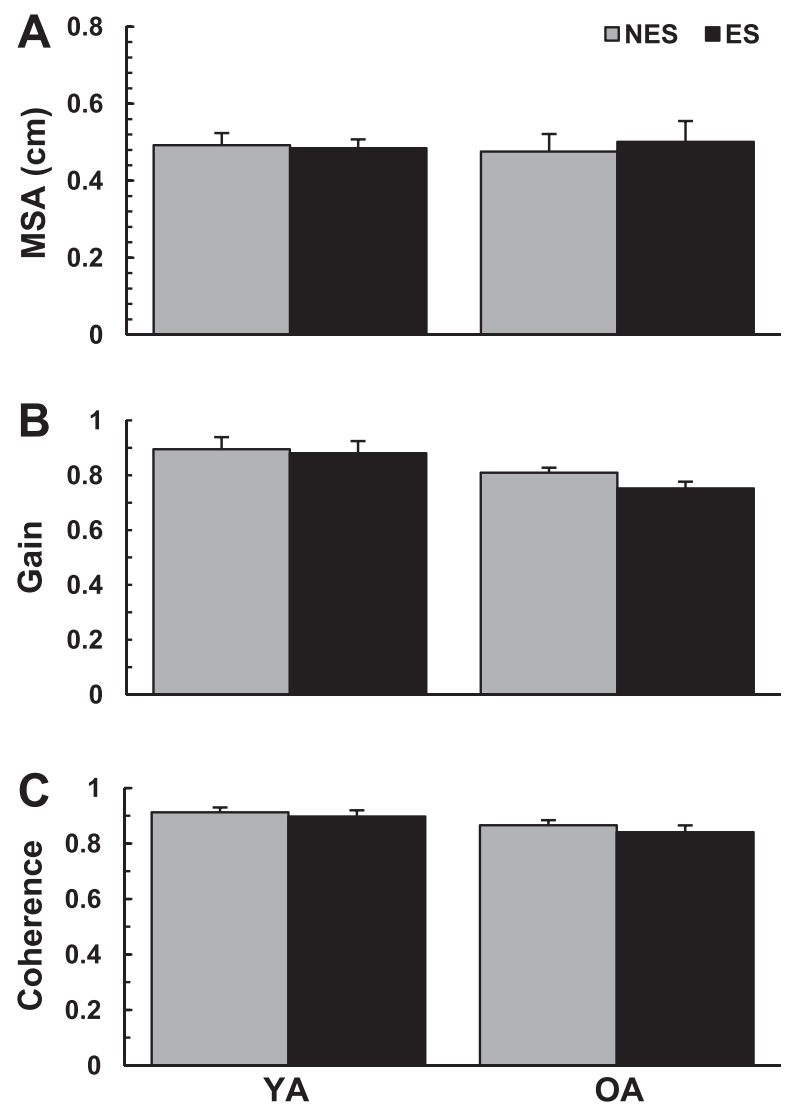

Fig. 5. Means and standard errors of postural control variables assessed during the Simple Room Movement condition. Data are presented for both age groups (YA - young adults, OA - older adults) and both conditions (NES - No Electrical Stimulation, ES - Electrical Stimulation). MSA - Mean sway amplitude in the anterior-posterior direction.

stimulation is used for each subject. The search for optimal individual stimulation intensities was not adopted in this work because this procedure would have prolonged significantly the duration of the experiment, causing tiredness, especially in older adults, which could have influenced their performance. We adopted as stimulus intensity $90 \%$ of the individual sensory threshold based on previous studies that showed that this intensity is effective in attenuating postural sway in comparison to the condition with zero noise-level stimulation (Priplata et al., 2006; Magalhaes and Kohn, 2012; Dettmer et al., 2015).

\section{SR effects on postural control}

The results showed that subsensory electrical noise led to decreased postural sway depending on the task demand, i.e., with unpredictable complex movement of the visual field. We expected that young adults would improve postural control with the application of SR stimulation only in more demanding tasks and our results confirmed this hypothesis. No significant SR effect in postural stability was obtained for the condition with eyes closed, however sway parameters in older adults tended toward 

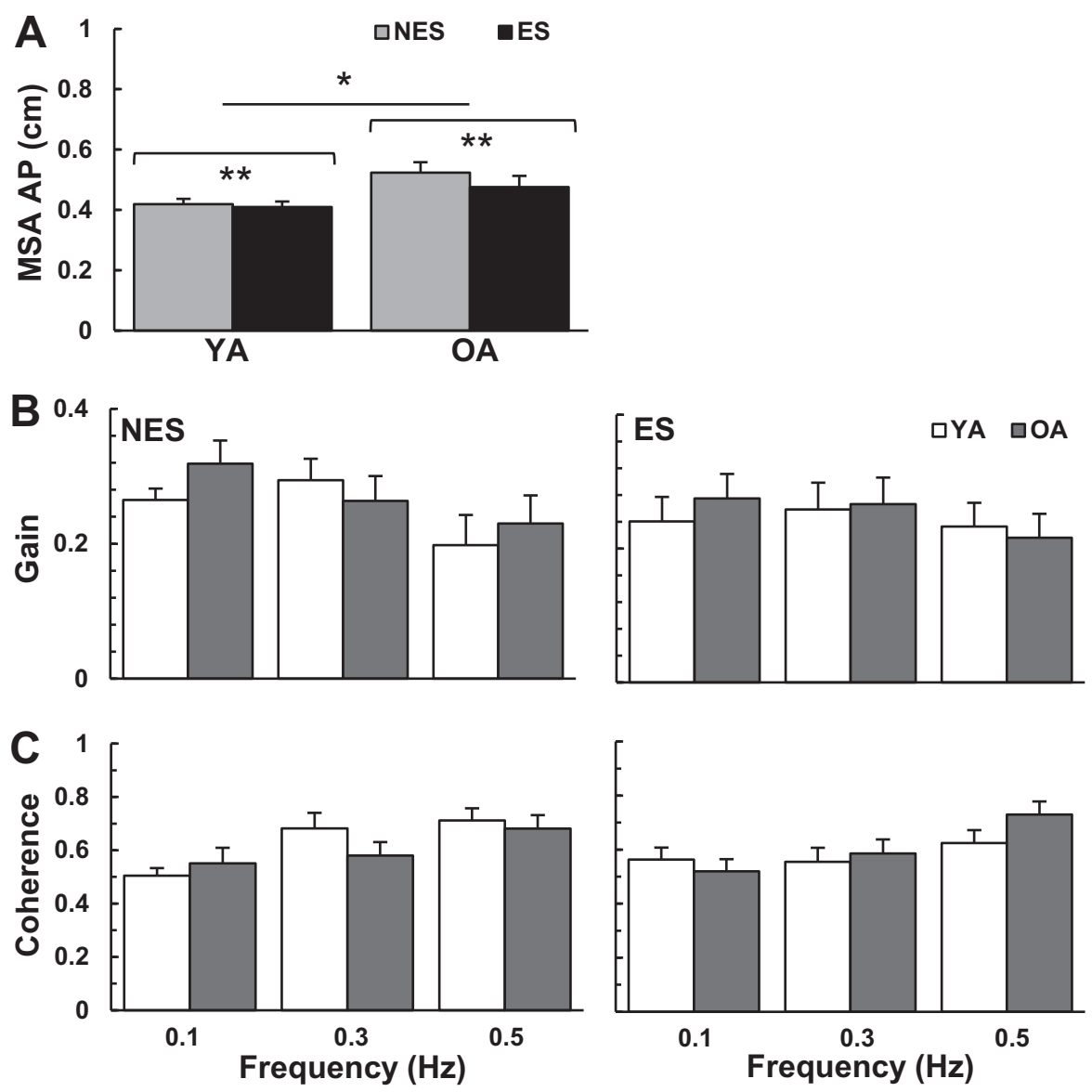

Fig. 6. Means and standard errors of postural control variables assessed during the Complex Room Movement condition. Data are presented for both age groups (YA - young adults, OA - older adults) and both conditions (NES - No Electrical Stimulation, ES - Electrical Stimulation). Gain and Coherence data are presented for each frequency that composed the room movement. MSA - Mean sway amplitude in the anterior-posterior direction. "Significant differences between the age groups $(P<0.05)$. ${ }^{* *}$ Significant differences between the conditions $(P<0.05)$.

those of young adults when SR stimulation was added. Whereas the interaction analysis showed age-related significant differences only without noise stimulation, inter-individual variability may have accounted for the non-significant main age effect in this condition. Conversely, previous studies reported that mechanical SR stimulation during upright stance with eyes closed can be significantly more effective in older adults in comparison to young adults (Priplata et al., 2002; Wu et al., 2007; Dettmer et al., 2015). Besides the different postural measures adopted in these different studies, it is possible that individual adjustments of noise stimulation are less crucial for obtaining significant effects when mechanical noise is used, in comparison to electrical noise. Furthermore the nature of the stimulus itself (mechanical versus electrical) may contribute to the different findings.

For the sensory conflict conditions, similar results between the age groups were observed, with both groups showing no SR effects for the simple movement condition and increased stability with the addition of SR stimulation for the complex movement condition, in comparison to the NES condition. Despite the larger sway amplitude observed in older participants in the complex condition, this group was not significantly more affected by electrical noise stimulation than young adults were. Different outcomes between conditions with respect to SR effects might be related to the task demand, in which specific reweighting processes may be involved in each situation (Prioli et al., 2006; Toledo and Barela, 2014). In this sense, improvement of proprioception feedback seems to be relevant for postural stability depending on the importance attributed to this sensory subsystem in comparison to other sensory sources (e.g., vision). When dealing with a simple periodic movement of the visual field, the strong coupling of postural oscillations to the visual manipulation (gain and coherence values close to 0.9 ) suggests that, in this particular condition, visual information was more relevant than the other systems for controlling posture. It seems that the postural control system decreases the relative weight of proprioceptive inputs due to an exquisite sensitivity to periodic optic flows. In contrast, during unpredictable room movement, there could have been a saturation of the postural response to the increased frequency content and peakto-peak amplitude of the optic flow (Peterka and Benolken, 1995), resulting in an increased relative importance of proprioceptive and vestibular inputs.

Moreover, the SR effects on sway amplitude but not on gain and coherence variables observed in the CM 
condition indicated that body sway was reduced due to the application of noise stimulation, but was still influenced by the visual manipulation. Absence of significant coherence at the lowest frequency of room movement $(0.1 \mathrm{~Hz})$ was also observed for this condition. This is consistent with previous research results (Dijkstra et al., 1994; Musolino et al., 2006) which suggested that postural sway is more sensitive to higher optical flow frequencies.

A further rationale behind task-specificity results could be the amount of attention directed onto the proprioceptive system. Earlier electrophysiological findings evidenced that directing attentional focus to the task increases the speed of proprioceptive stimulus evaluation (Kida et al., 2003; Toledo et al., 2016a,b). In our previous studies we have provided evidence of increased cognitive effort (augmented event-related desynchronization) in both young and older adults in order to respond faster to the kinesthetic perception (Toledo et al., 2016a,b). This represents an important strategy, especially in older adults, who need to compensate for somatosensory loss (e.g., delayed stimulus arrival at the cortex) as well as cognitive deficits (e.g., reduced selective attention). Furthermore, the rate of muscle spindle firing was shown to be modulated by attention in humans (Hospod et al., 2007). According to these findings, greater proprioceptive acuity could be provided by changes in the fusimotor control when attention was selectively directed onto proprioceptive recognition. In this regard, the condition with complex room movement might be more sensitive to small proprioceptive signaling changes in comparison to the other conditions also due to an increased attentional involvement. In fact, several subjects from both groups suspected that a visual manipulation occurred during the complex movement, but not during the simple movement, although this feeling was mostly described as a self-motion perception. The assumption of the influence of attention on the reweighting process is in accordance with a previous study of ours, which showed that body sway is differently controlled based on the awareness about the room movement (Barela et al., 2009).

In the present study, we have manipulated the visual system to cause a sensory conflict between the various sensory inputs and observed how stochastic resonance of the proprioceptive system could help compensate such manipulation. A different protocol could manipulate the proprioceptive system and investigate if SR effects could improve the performance of either of the two other main sensory subsystems (vision or vestibular feedback). Even though the contribution of the vestibular system to postural control can be noisy and weak during quiet or mildly perturbed stance (Mergner et al., 1993; Fitzpatrick and McCloskey, 1994), significant effects of stochastic galvanic vestibular stimulation on postural control were observed by recent studies with healthy young adults (Mulavara et al., 2011; Goel et al., 2015) as well as in individuals with Parkinson's disease (Pal et al., 2009; Samoudi et al., 2015). We suggest that SR stimulation at the vestibular level could also improve the resolution of the sensory conflicting situation generated by room movement and improve postural stability. Furthermore, postural sway could be reduced even more with a combination of electrical noise stimulation of two or more sensory systems.

\section{Non-significant correlation between proprioceptive and postural measures}

The non-significant correlation between response time and body sway observed in this study indicates that the effectiveness of the application of noise during the postural task (decreased MSA during CM condition) could not be estimated from the proprioceptive functional results (reduced RT). This finding could be attributed to methodological issues, especially with respect to differences in the body configuration between the tasks. Previous studies considered that active fusimotor drive (via gamma efferent system) could improve the quality of afferent information originated from muscle spindles (Bergenheim et al., 1995; Tock et al., 2005). Accordingly, increased asynchronous activity of extrafusal muscle fibers would increase the sensitivity of intrafusal fibers (Fallon et al., 2004), presumably acting as an intrinsic source of noise controlling for SR of the proprioceptive system. If so, a combination of internal and external noises $(\alpha-\gamma$ co-activation during muscle contraction and external electrical noise stimulation, respectively) that occurred during upright stance might improve afferent proprioceptive signaling differently from the situation without muscular contraction (proprioceptive assessment). Furthermore, the proprioceptive assessment was performed with the leg flexed, i.e., the gastrocnemius muscles were disengaged. Under this condition, afferent signaling from the triceps surae might be attenuated in comparison to the extended leg case. Apart from body configuration, other factors could be also considered, such as the cognitive involvement in each task and the parameters for electrical stimulation. The indirect effects of SR on force control is thought to be dependent on the frequency spectrum of the noise (Trenado et al., 2014), which could be task specific.

\section{Clinical implications}

This study showed that a sample of a population with decreased kinesthetic perception (higher response times to perception of ankle rotation) related to aging can benefit from the application of an external noise stimulus. The subsensory stimulation is supposed to facilitate the transmission of information on the positioning of body segments. Since the mean decrease in response times for the older group was $120 \mathrm{~ms}$, this should result in improvements in balance recovery and in avoiding falls. In addition to earlier responses, improved quality of sensory signaling and processing with SR would contribute to the effectiveness of sensorimotor processing, leading to selection of appropriate motor response. However, it is questionable whether functional benefits would be also obtained in realistic environmental conditions, which have several distracters and physical constraints. Dettmer and colleagues (2016) recently showed that postural stability 
in a group of older adults was unchanged by mechanical noise applied through insoles (Dettmer et al., 2016). On the other hand, Lipsitz et al. (2015) showed that vibratory noise applied to the sole of the foot improved the performance in more dynamic tasks (i.e. timed up-and-go and gait) in older adults (Lipsitz et al., 2015). The current study showed that subsensory electrical stimulation over the legs can increase postural stability during quiet standing depending on the sensory situation. Thus, further studies are needed to determine the most appropriate parameters and stimulation sites that result in balance improvements in more realistic situations. Furthermore, the duration of the effects and habituation also need to be investigated. Once these aspects are well established, fragile subjects could benefit from noise stimulation applied by electrodes fixed directly on specific body segments or applied through assistive devices (e.g. canes, walkers) and insoles. For those impaired individuals who adhere to training and rehabilitation programs, advantage from SR stimulation approaches could be even more relevant. Indeed, the combination of electrical noise stimulation with functional rehabilitation training has been shown to optimize the improvement of posture control in subjects with ankle instability, compared to functional training without stimulation (Ross et al., 2007). Studies in this aspect may help not only aged individuals and patients with degenerative diseases to benefit more from therapies, but could also optimize sport training gains of young athletes.

\section{CONCLUSIONS}

The results of the current study provide evidence that proprioceptive function can be improved in both young and older adults with the application of subsensory electrical noise. Enhancement of kinesthetic perception and increased postural stability are believed to be related to improved encoding of afferent information by sensory neurons when the electrical noise is applied on the lower limbs. The noise stimulation may act not only on peripheral receptors, but also on neurons located at higher levels of the central nervous system (which receive and process the activity evoked on axons of the peripheral nervous system by the stimulation), contributing to better signaling, transmission and processing of sensory information originating from the ankle joint. However, the functional findings of this study are still premature to indicate practical applications of this stimulation method for improving somatosensory function in daily life. Further investigations should consider the task-specificity effectiveness of stochastic resonance stimulation, as well as the optimum stimulation parameters for more substantial functional improvements.

\section{CONFLICT OF INTEREST}

The authors declare that there are no conflicts of interest.

Acknowledgments-This research was funded by Grants from FAPESP - Brazil [grant no. 2011/17193-0] and CNPq - Brazil [grant no. 303809/2016-7]. DRT received a PhD scholarship [grant no. 2009/09286-9] and holds a Post-Doctoral Grant [grant no. 2013/14667-7], both from FAPESP.

\section{REFERENCES}

Aminzadeh F, Edwards N (1998) Exploring seniors' views on the use of assistive devices in fall prevention. Public Health Nurs 15:297-304.

Barela AM, Barela JA, Rinaldi NM, de Toledo DR (2009) Influence of imposed optic flow characteristics and intention on postural responses. Mot Control 13:119-129.

Barela JA, Genoves GG, Alleoni B, Barela AM (2013) Visual reweighting in postural control is less adaptative in older adults. Health 5:74-79.

Bergenheim M, Johansson H, Pedersen J (1995) The role of the gamma-system for improving information transmission in populations of la afferents. Neurosci Res 23:207-215.

Borel L, Ribot-Ciscar E (2016) Improving postural control by applying mechanical noise to ankle muscle tendons. Exp Brain Res 234:2305-2314.

Collins AT, Blackburn JT, Olcott CW, Dirschl DR, Weinhold PS (2009) The effects of stochastic resonance electrical stimulation and neoprene sleeve on knee proprioception. J Orthop Surg Res $4: 3$.

Collins JJ, Imhoff TT, Grigg P (1996) Noise-enhanced tactile sensation. Nature 383:770.

Cordo P, Inglis JT, Verschueren S, Collins JJ, Merfeld DM, Rosenblum S, Buckley S, Moss F (1996) Noise in human muscle spindles. Nature 383:769-770.

Dettmer M, Pourmoghaddam A, Lee BC, Layne CS (2015) Effects of aging and tactile stochastic resonance on postural performance and postural control in a sensory conflict task. Somatosens Mot Res 32:128-135.

Dettmer M, Pourmoghaddam A, Lee BC, Layne CS (2016) Do aging and tactile noise stimulation affect responses to support surface translations in healthy adults? Curr Gerontol Geriatr Res 2016:2941964.

Dijkstra TM, Schoner G, Giese MA, Gielen CC (1994) Frequency dependence of the action-perception cycle for postural control in a moving visual environment: relative phase dynamics. Biol Cybern 71:489-501.

Fallon JB, Carr RW, Morgan DL (2004) Stochastic resonance in muscle receptors. J Neurophysiol 91:2429-2436.

Fitzpatrick R, McCloskey DI (1994) Proprioceptive, visual and vestibular thresholds for the perception of sway during standing in humans. J Physiol 478(Pt 1):173-186.

Genoves GG, Barela AM, Sanches C, Barela JA (2016) Attentional artifacts in sensorimotor coupling in the postural control of young adults. Exp Brain Res 234:3641-3647.

Goel R, Kofman I, Jeevarajan J, De Dios Y, Cohen HS, Bloomberg JJ, Mulavara AP (2015) Using low levels of stochastic vestibular stimulation to improve balance function. PLoS One 10:e0136335.

Gravelle DC, Laughton CA, Dhruv NT, Katdare KD, Niemi JB, Lipsitz LA, Collins JJ (2002) Noise-enhanced balance control in older adults. Neuroreport 13:1853-1856.

Hospod V, Aimonetti JM, Roll JP, Ribot-Ciscar E (2007) Changes in human muscle spindle sensitivity during a proprioceptive attention task. J Neurosci 27:5172-5178.

Jeka JJ, Oie KS, Kiemel T (2008) Asymmetric adaptation with functional advantage in human sensorimotor control. Exp Brain Res 191:453-463.

Keshner EA, Slaboda JC, Day LL, Darvish K (2014) Visual conflict and cognitive load modify postural responses to vibrotactile noise. J Neuroeng Rehabil 11:6.

Kida T, Nishihira Y, Hatta A, Wasaka T, Nakata H, Sakamoto M, Nakajima T (2003) Changes in the somatosensory N250 and P300 by the variation of reaction time. Eur J Appl Physiol 89:326-330. 
Kimura T, Kouzaki M (2013) Electrical noise to a knee joint stabilizes quiet bipedal stance. Gait Posture 37:634-636.

Lipsitz LA, Lough M, Niemi J, Travison T, Howlett H, Manor B (2015) A shoe insole delivering subsensory vibratory noise improves balance and gait in healthy elderly people. Arch Phys Med Rehabil 96:432-439.

Lord SR, Ward JA (1994) Age-associated differences in sensorimotor function and balance in community dwelling women. Age Ageing 23:452-460.

Lugo E, Doti R, Faubert J (2008) Ubiquitous crossmodal Stochastic Resonance in humans: auditory noise facilitates tactile, visual and proprioceptive sensations. PLoS One 3:e2860.

Magalhaes FH, Kohn AF (2011) Vibratory noise to the fingertip enhances balance improvement associated with light touch. Exp Brain Res 209:139-151.

Magalhaes FH, Kohn AF (2012) Imperceptible electrical noise attenuates isometric plantar flexion force fluctuations with correlated reductions in postural sway. Exp Brain Res 217:175-186.

Magalhaes FH, Kohn AF (2014) Effectiveness of electrical noise in reducing postural sway: a comparison between imperceptible stimulation applied to the anterior and to the posterior leg muscles. Eur J Appl Physiol 114:1129-1141.

Manjarrez E, Mendez I, Martinez L, Flores A, Mirasso CR (2007) Effects of auditory noise on the psychophysical detection of visual signals: cross-modal stochastic resonance. Neurosci Lett 415:231-236.

McChesney JW, Woollacott MH (2000) The effect of age-related declines in proprioception and total knee replacement on postural control. J Gerontol A Biol Sci Med Sci 55:M658-M666.

McDonnell MD, Abbott D (2009) What is stochastic resonance? Definitions, misconceptions, debates, and its relevance to biology. PLoS Comput Biol 5:e1000348.

Mendez-Balbuena I, Huidobro N, Silva M, Flores A, Trenado C, Quintanar L, Arias-Carrion O, Kristeva R, Manjarrez E (2015) Effect of mechanical tactile noise on amplitude of visual evoked potentials: multisensory stochastic resonance. J Neurophysiol 114:2132-2143.

Mendez-Balbuena I, Manjarrez E, Schulte-Monting J, Huethe F, Tapia JA, Hepp-Reymond MC, Kristeva R (2012) Improved sensorimotor performance via stochastic resonance. J Neurosci 32:12612-12618.

Mergner T, Hlavacka F, Schweigart G (1993) Interaction of vestibular and proprioceptive inputs. J Vestib Res 3:41-57.

Moss F, Ward LM, Sannita WG (2004) Stochastic resonance and sensory information processing: a tutorial and review of application. Clin Neurophysiol 115:267-281.

Mulavara AP, Fiedler MJ, Kofman IS, Wood SJ, Serrador JM, Peters B, Cohen HS, Reschke MF, Bloomberg JJ (2011) Improving balance function using vestibular stochastic resonance: optimizing stimulus characteristics. Exp Brain Res 210:303-312.

Musolino MC, Loughlin PJ, Sparto PJ, Redfern MS (2006) Spectrally similar periodic and non-periodic optic flows evoke different postural sway responses. Gait Posture 23:180-188.

Pal S, Rosengren SM, Colebatch JG (2009) Stochastic galvanic vestibular stimulation produces a small reduction in sway in Parkinson's disease. J Vestib Res 19:137-142.

Peterka RJ, Benolken MS (1995) Role of somatosensory and vestibular cues in attenuating visually induced human postural sway. Exp Brain Res 105:101-110.
Prioli AC, Cardozo AS, de Freitas Junior PB, Barela JA (2006) Task demand effects on postural control in older adults. Hum Mov Sci 25:435-446.

Priplata A, Niemi J, Salen M, Harry J, Lipsitz LA, Collins JJ (2002) Noise-enhanced human balance control. Phys Rev Lett 89:238101.

Priplata AA, Niemi JB, Harry JD, Lipsitz LA, Collins JJ (2003) Vibrating insoles and balance control in elderly people. Lancet 362:1123-1124.

Priplata AA, Patritti BL, Niemi JB, Hughes R, Gravelle DC, Lipsitz LA, Veves A, Stein J, Bonato P, Collins JJ (2006) Noise-enhanced balance control in patients with diabetes and patients with stroke. Ann Neurol 59:4-12.

Ribot-Ciscar E, Hospod V, Aimonetti JM (2013) Noise-enhanced kinaesthesia: a psychophysical and microneurographic study. Exp Brain Res 228:503-511.

Rosenberg JR, Halliday DM, Breeze P, Conway BA (1998) Identification of patterns of neuronal connectivity-partial spectra, partial coherence, and neuronal interactions. J Neurosci Methods 83:57-72.

Ross SE, Arnold BL, Blackburn JT, Brown CN, Guskiewicz KM (2007) Enhanced balance associated with coordination training with stochastic resonance stimulation in subjects with functional ankle instability: an experimental trial. J Neuroeng Rehabil 4:47.

Samoudi G, Jivegard M, Mulavara AP, Bergquist F (2015) Effects of stochastic vestibular galvanic stimulation and LDOPA on balance and motor symptoms in patients with Parkinson's disease. Brain Stimul 8:474-480.

Tabachnick BG, Fidell LS (2007) Using multivariate statistics. Boston: Pearson Education.

Tinetti ME, Kumar C (2010) The patient who falls: "It's always a tradeoff". JAMA 303:258-266.

Tock Y, Inbar GF, Steinberg Y, Ljubisavljevic M, Thunberg J, Windhorst U, Johansson H (2005) Estimation of muscle spindle information rate by pattern matching and the effect of gamma system activity on parallel spindles. Biol Cybern 92:316-332.

Toledo DR, Barela JA (2010) Sensory and motor differences between young and older adults: somatosensory contribution to postural control. Rev Bras Fisioter 14:267-275.

Toledo DR, Barela JA (2014) Age-related differences in postural control: effects of the complexity of visual manipulation and sensorimotor contribution to postural performance. Exp Brain Res 232:493-502.

Toledo DR, Barela JA, Manzano GM, Kohn AF (2016a) Age-related differences in EEG beta activity during an assessment of ankle proprioception. Neurosci Lett 622:1-5.

Toledo DR, Manzano GM, Barela JA, Kohn AF (2016b) Cortical correlates of response time slowing in older adults: ERP and ERD/ERS analyses during passive ankle movement. Clin Neurophysiol 127:655-663.

Trenado C, Mikulic A, Manjarrez E, Mendez-Balbuena I, SchulteMonting J, Huethe F, Hepp-Reymond MC, Kristeva R (2014) Broad-band Gaussian noise is most effective in improving motor performance and is most pleasant. Front Hum Neurosci 8:22.

Volgushev M, Eysel UT (2000) Neuroscience. Noise makes sense in neuronal computing. Science 290:1908-1909.

Wu F, Wang R, Jin S, Hu X, Yang Y, Zhang J, Youshimura N (2007) Effect of noise-enhanced on the balance control ability in older adults. In: Duffy VG, editor. ICDHM 2007 Lecture Notes in Computer Science, Digital Human Modeling, Vol. 4561. Berlin, Heidelberg: Springer. p. 483. 